\title{
UNA MEDIDA DE LA INCERTIDUMBRE BASADA EN LA ENTROPÍA PARA CUANTIFICAR EL IMPAC- TO DE LA PÉRDIDA DE INFORMACIÓN DE LOS SISTEMAS DE GESTIÓN
}

\author{
Max Guillermo Schwarz Diaz
}

\section{Resumen}

El presente artículo plantea una medida para cuantificar la incertidumbre generada por la pérdida de información causada por la ausencia parcial de componentes en los sistemas de gestión en comparación con la existencia de un modelo ideal de información completa, haciendo uso de una estimación basada en la entropía de Shannon, de la teoría matemática de la información, que puede ser utilizada para medir la entropía de cualquier modelo de gestión. Este articulo abarca un aspecto del proceso de investigación doctoral del autor para la construcción de un modelo instrumental de sistema integrado de gestión de baja entropía que mejora la efectividad de la organización.

Palabras clave: Sistema de Gestión, Entropía, Medida de Entropía, Entropía de Shannon, Función de pérdida de Taguchi, Sistemas de pesos.

\section{Abstract}

This paper proposes a measure to quantify the uncertainty caused by the loss of information caused by the partial absence of components in systems management compared to the existence of an ideal model of complete information, using an estimate based on the entropy Shannon's mathematical theory of information that can be used to measure the entropy of any model management. This paper deals with one aspect of the process of doctoral research by the author to 
construct an instrumental model of integrated management system low entropy that improves the effectiveness of the organization.

Keyword: Management System, Entropy, Entropy measure, Shannon entropy, Taguchi Loss Function, Systems pesos.

\section{INTRODUCCIÓN}

El presente trabajo plantea una medida para cuantificar la incertidumbre generada por la pérdida de información causada por la ausencia parcial de componentes en los sistemas de gestión haciendo uso de una estimación basada en la entropía de Shannon, que puede ser utilizada para medir la entropía de cualquier modelo de gestión. De igual forma, se demuestra mediante la función de pérdida de calidad de Taguchi, la convergencia de los pesos relativos de los componentes del modelo hacia una configuración de pesos ideal que proporciona la menor entropía posible.

\section{REVISIÓN DE LA LITERATURA}

Los primeros trabajos de entropía de información basados en la teoría de Shannon (Frizelle \& Woodcock, 1995), aplicados a los sistemas de gestión fueron realizados por Frizelle y Woodcock (Isik, 2010). en 1995. El trabajo planteaba una medida de la complejidad de los sistemas manufacturados y de la contribución de cada fuente operacional. Los sistemas manufacturados se asumian formados por una cantidad finita de estados y los autores propusieron dos medidas de complejidad, una estructural y otra operacional, siendo estas una ponderación apropiada de las entropías de información sobre los estados definidos previamente. Luego, en el año 2010, Isik (Kannan, \& et al, 2008) mejora esas medidas y las aplica a la medida de la complejidad de la cadena de suministros. En este trabajo, las medidas estructural y operacional provienen de una entropía modificada por un factor de desviación de los valores esperados de los recursos en cada estado del sistema.

\section{ESTRUCTURA DE PESOS}

Requerimos determinar una estructura de pesos para cada componente de manera tal que reflejen su importancia relativa en términos de resultados de gestión y aporte de información. Estos valores no serán absolutos, lo que en realidad tiene importancia es la proporcionalidad entre ellos, que reflejan las relaciones de niveles de gestión y nos permitirán hacer un cálculo de entropía que, por tanto, refleje las relaciones de superioridad entre ellos. Además, con el fin de simplificar los cálculos y obtener esta secuencia de pesos, notamos que hay componentes del Sistema Integrado de Gestión que aportan casi lo mismo al sistema. Luego, en vez de calcular 20 valores de gestión calcularemos solo 5 , agrupándolos de la siguiente manera:

Grupo 1 (4): Política; Requisitos del Cliente, legales y regulatorios; Revisión Gerencial y Creatividad e Innovación. 
Grupo 2 (1): Aspectos Especializados.

Grupo 3 (2): Objetivos y Metas y Control Operacional.

Grupo 4 (2): No Conformidad, Acción Preventiva y Correctiva; Aprendizaje y Crecimiento.

Grupo 5 (11): Los demás componentes.

Abstraemos el modelo de Sistema Integrado como un sistema único $\mathrm{y}$ analizamos la "calidad de la gestión" del sistema en base a la función de pérdida de calidad de Taguchi (Liu \& Zhang, 2011), considerando estos cinco grupos como las entradas. Esta abstracción va de acuerdo con nuestros fines de obtener el mejor modelo, que en este caso es el que gestiona mejor o tiene mejor calidad de gestión. La función de Taguchi viene dada por:

$$
L(y)=K(y-m)^{2}
$$

Donde:

$L(y)$ : Costo de penalidad. Pérdida en dinero por unidad.

$K \quad$ : Constante de proporcionalidad que depende de la importancia financiera de la variable de calidad

y : Característica funcional del producto o variable calidad

$m$ : Valor nominal de la variable $y$

Para nuestro caso y representa cada grupo como un producto del sistema (modelo) que tiene un objetivo de calidad $\mathrm{m}$, con una importancia (aporte a la gestión) $\mathrm{K}$ en la gestión de calidad del Sistema y que, según Taguchi, produce una pérdida de calidad en la gestión $\mathrm{L}(\mathrm{y})$.
Según esto, si el vector representa los pesos de calidad (en la gestión) que aportan los grupos 1,2,3,4 y 5 respectivamente, entonces la función de pérdida de Taguchi, para nuestro sistema, tendría la siguiente forma:

$$
\overrightarrow{L(p)}=K_{1}\left(p_{1}-m_{1}\right)^{2}+K_{2}\left(p_{2}-m_{2}\right)^{2}+K_{3}\left(p_{3}-m_{3}\right)^{2}+K_{4}\left(p_{4}-m_{4}\right)^{2}+K_{5}\left(p_{5}-m_{5}\right)^{2}
$$

(2)

De esta manera, el paso siguiente consiste en elegir los valores de importancia y los objetivos de calidad de las variables. En esta parte se deja notar la significancia relativa, más no absoluta, de los pesos encontrados eligiendo valores de $\mathrm{K}$ y $\mathrm{m}$ que representen las relaciones entre los grupos 1, 2, 3, 4 y 5 , sin dar tanta importancia a los valores absolutos.

- $\mathrm{p}_{2}$ es el componente más importante, por lo tanto, el grupo 2 tendrá un aporte de calidad (en la gestión) mayor que los demás. $p_{2}>p_{i}, i=1,3,4,5$. E1 segundo componente más importante es $\mathrm{p}_{1}$, por tanto $p_{1}>p_{3}, p_{1}>p_{4}$.

- Los grupos 3 y 4 están presentes en muchos Sistemas de Gestión estandarizados $\mathrm{y}$, en ausencia del grupo 2, juntos heredan su funcionalidad, luego, se debe tener $K_{2}=K_{3}+K_{4}$. Juntando el grupo 3 y el grupo 5 se obtienen medios suplentes para que el sistema busque la excelencia y mejora continua, tal como lo hace el grupo 1. Luego, se debe tener $K_{1}=K_{3}+K_{5}$ 
- Se tiene 20 componentes presentes en la gestión que se ha distribuido en 5 grupos pi; la suma ponderada de estos grupos es igual a la unidad.

$$
\begin{aligned}
& 4 p_{1}+p_{2}+2 p_{3}+2 p_{4}+11 p_{5}=1 \\
& \sum_{i=1}^{5} a_{i} p_{i}=\vec{a} \cdot \vec{p}=1 \\
& \vec{a}=(4,1,2,2,11)
\end{aligned}
$$

- Para poder estimar los valores de los pesos pi se usará simulaciones Montecarlo, donde las variables $\mathrm{K}_{3}, \mathrm{~K}_{4}, \mathrm{~K}_{5}$, mi serán variables aleatorias y el modelo será el minimizar la función de Taguchi con las restricciones descritas arriba para cada valor escogido aleatoriamente de dichas variables.

- La variable mi es un valor nominal, donde se hace mínimo la función de Taguchi del grupo i sin restricción alguna. Entonces, en ese caso, representaría el valor del peso del grupo i a la gestión, por tanto: $\mathrm{mi}<1, \mathrm{i}=1,2,3,4,5$.

- Si resolvemos el problema de minimización de la ecuación (2) sujeta solo a la restricción de la ecuación (3) por medio de los multiplicadores de Lagrange, obtenemos que la variable $\mathrm{Ki}$ no es significativa en la solución y no importa que valores pueda tomar esta variable (ver anexo). Entonces simularemos arbitrariamente valores de $0<k_{i}<150$,

y se verá que no es significativo en el resultado obtenido.

Finalmente, el problema a minimizar queda como:

$$
\begin{array}{ll}
\vec{L}(\vec{p})= & K_{1}\left(p_{1}-m_{1}\right)^{2}+K_{2}\left(p_{2}-m_{2}\right)^{2}+K_{3}\left(p_{3}-m_{3}\right)^{2}+K_{4}\left(p_{4}-m_{4}\right)^{2}+K_{5}\left(p_{5}-m_{5}\right)^{2} \\
\text { s.a } & 4 p_{1}+p_{2}+2 p_{3}+2 p_{4}+11 p_{5}=1 \\
& p_{1}>0, i=1,2,3,4,5 \\
& p_{2}>p_{1}>p_{3} \\
& p_{1}>p_{4}, p_{2}>p_{5}
\end{array}
$$

Este problema matemático lo resolveremos usando el algoritmo SQP (Sequential Quadratic Programming). Donde se ha simulado 105 eventos. Como resolveremos el problema numéricamente, la restricción pi>0 no es suficiente; entonces, solo consideraremos válidos los eventos donde pi>104. Los resultados de la simulación para los valores de pi se pueden apreciar en los histogramas de la Figura 1. Vemos claramente que los valores de $\mathrm{p}_{1}, \mathrm{p}_{2}, \mathrm{p}_{3}, \mathrm{p}_{4}$ están restringidos y están centrados; para la estimación de estos valores, tomamos el valor esperado es estas 04 variables. Entonces:

$p_{1}=0.0808, p_{2}=0.2080, p_{3}=0.0628, p_{4}=0.0629$

Lo que lleva a concluir que $p_{5}=0.0197$. Con estos valores, ya podemos hacer un cálculo de la entropía. El cuadro 1 muestra la lista de pesos finales. 


\begin{tabular}{|c|c|c|c|c|}
\hline Componente & Notación & \begin{tabular}{|c|} 
Peso en la Gestón \\
por el modelo \\
propuesto
\end{tabular} & \begin{tabular}{|c|} 
Pesos empi- \\
ricos en la \\
literatura \\
\end{tabular} & $\begin{array}{l}\text { Referencias } \\
\text { del peso de } \\
\text { la Gestión }\end{array}$ \\
\hline Política & $x_{1}$ & $8.08 \%(0.0808)$ & 0.10 & {$[12,13,14]$} \\
\hline $\begin{array}{l}\text { Aspectos Especiali- } \\
\text { zados }\end{array}$ & $x_{2}$ & $20.80 \%(0.2080)$ & 0.20 & {$[13,14]$} \\
\hline $\begin{array}{l}\text { Requisitos del cliente, } \\
\text { legales y regulatorios. }\end{array}$ & $x_{3}$ & $8.08 \%(0.0808)$ & 0.10 & {$[15,16,17]$} \\
\hline Objetivos y Metas & $x_{4}$ & $6.28 \%(0.0628)$ & 0.05 & {$[13,14]$} \\
\hline Programas de Gestión & $x_{5}$ & $1.97 \%(0.0197)$ & 0.02 & {$[13,14]$} \\
\hline $\begin{array}{l}\text { Estructura y Respon- } \\
\text { sabilidad }\end{array}$ & $x_{6}$ & $1.97 \%(0.0197)$ & 0.02 & {$[12,18,19,20]$} \\
\hline $\begin{array}{l}\text { Competencia, entre- } \\
\text { namiento y capaci- } \\
\text { tación }\end{array}$ & $x_{7}$ & $1.97 \%(0.0197)$ & 0.02 & {$[12,13,14]$} \\
\hline Comunicaciones & $x_{8}$ & $1.97 \%(0.0197)$ & 0.02 & {$[13,14]$} \\
\hline Documentación & $x_{9}$ & $1.97 \%(0.0197)$ & 0.02 & {$[13,14]$} \\
\hline $\begin{array}{l}\text { Control de Documen- } \\
\text { tos }\end{array}$ & $x_{10}$ & $1.97 \%(0.0197)$ & 0.02 & {$[13,14]$} \\
\hline Control Operacional & $x_{11}$ & $6.28 \%(0.0628)$ & 0.05 & {$[13,14]$} \\
\hline $\begin{array}{l}\text { Respuesta a emer- } \\
\text { gencias }\end{array}$ & $x_{12}$ & $1.97 \%(0.0197)$ & 0.02 & {$[13,14]$} \\
\hline $\begin{array}{l}\text { Seguimiento, monito- } \\
\text { reo y Medición }\end{array}$ & $x_{13}$ & $1.97 \%(0.0197)$ & 0.02 & {$[13,14]$} \\
\hline $\begin{array}{l}\text { No conformidad, ac- } \\
\text { ción preventiva y cor- } \\
\text { rectiva }\end{array}$ & $x_{14}$ & $6.29 \%(0.0629)$ & 0.04 & {$[13,14]$} \\
\hline Control de registros & $x_{15}$ & $1.97 \%(0.0197)$ & 0.02 & {$[13,14]$} \\
\hline Auditoría interna & $x_{16}$ & $1.97 \%(0.0197)$ & 0.02 & {$[12,13,14]$} \\
\hline Revisión Gerencial & $x_{17}$ & $8.08 \%(0.0808)$ & 0.10 & {$[12,18,19,20]$} \\
\hline $\begin{array}{l}\text { Control de condicio- } \\
\text { nes anormales de } \\
\text { operación }\end{array}$ & $x_{18}$ & $1.97 \%(0.0197)$ & 0.02 & {$[13,14]$} \\
\hline $\begin{array}{l}\text { Aprendizaje y creci- } \\
\text { miento }\end{array}$ & $x_{19}$ & $6.29 \%(0.0629)$ & 0.04 & {$[21,22,14]$} \\
\hline $\begin{array}{l}\text { Creatividad e inno- } \\
\text { vación }\end{array}$ & $x_{20}$ & $8.08 \%(0.0808)$ & 0.1 & {$[21,22]$} \\
\hline Total & & $100 \%(1.0)$ & & \\
\hline
\end{tabular}

Cuadro 1. pesos relativos de las componentes 


DISTANCIA:

$$
\begin{aligned}
& x_{2}, x_{4}, x_{12}, x_{18}, x_{19}, x_{20} \\
& G=1-\sum_{k=1}^{6} d_{k}=0.5462 \\
& \mathrm{H}=-0.2080 \log _{2} 0.2080-0.0628 \log _{2} 0.0628- \\
& 0.0197 \log _{2} 0.0197-0.0197 \log _{2} 0.0197- \\
& 0.0629 \log _{2} 0.0629-0.0808 \log _{2} 0.0808- \\
& 0.273 \log _{2} 0.2731-0.2731 \log _{2} 0.2731=2.5122
\end{aligned}
$$

\section{Sistema BPI [9]}

DISTANCIA:

$x_{2}, x_{4}, x_{5}, x_{12}, x_{18}, x_{19}$

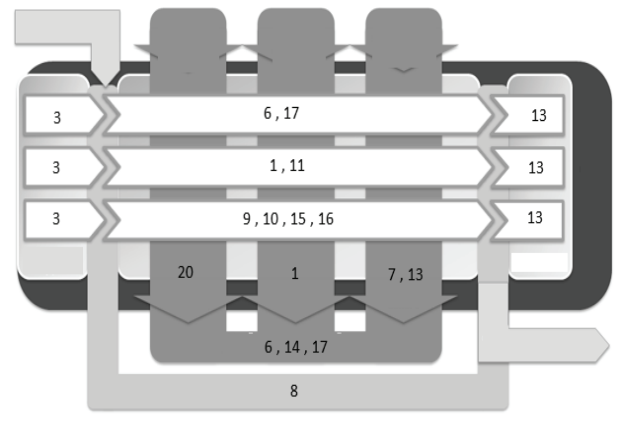

Figura 6. Modelo BPM

$G=1-\sum_{k=1}^{6} d_{k}=0.6072$

$\mathrm{H}=-0.2080 \log _{2} 0.2080-0.0628 \log _{2} 0.0628-$ $0.0197 \log _{2} 0.0197-0.0197 \log _{2} 0.0197$ $0.0197 \log _{2} 0.0197-0.0629 \log _{2} 0.0629$ $0.3036 \log _{2} 0.3036-0.3036 \log _{2} 0.3036=2.3521$

\section{Sistema MALCOM BALDRIGE [10]}

DISTANCIA:

$x_{2}, x_{4}, x_{5}, x_{12}, x_{16}, x_{18}$

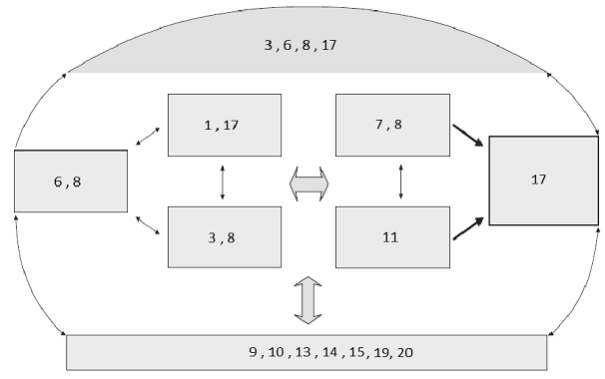

Figura 7. Modelo MALCOM BALDRIGE

$$
\begin{aligned}
G=1- & \sum_{k=1}^{6} d_{k}=0.6504 \\
\mathrm{H}=- & 0.2080 \log _{2} 0.2080-0.0628 \log _{2} 0.0628- \\
& 0.0197 \log _{2} 0.0197-0.0197 \log _{2} 0.0197- \\
& 0.0197 \log _{2} 0.0197-0.0197 \log _{2} 0.0197- \\
& 0.3252 \log _{2} 0.3252-0.3252 \log _{2} 0.3252=2.2225
\end{aligned}
$$

\section{Sistema APICS [11]}

DISTANCIA:

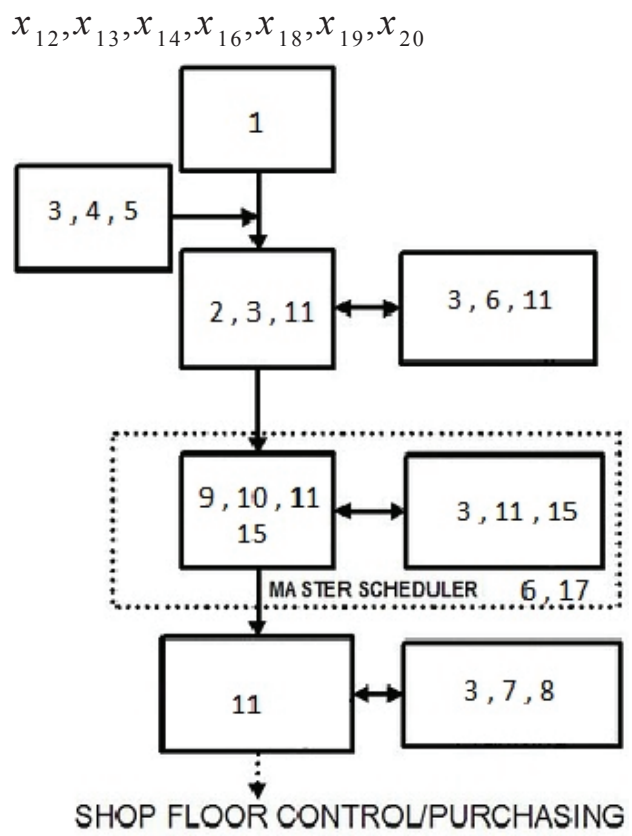

\section{Figura 8. Modelo APICS}

$$
\begin{aligned}
G=1- & \sum_{k=1}^{7} d_{k}=0.7146 \\
\mathrm{H}=- & 0.0197 \log _{2} 0.0197-0.0197 \log _{2} 0.0197- \\
& 0.0629 \log _{2} 0.0629-0.0197 \log _{2} 0.0197- \\
& 0.0197 \log _{2} 0.0197-0.0629 \log _{2} 0.0629- \\
& 0.0808 \log _{2} 0.0808-0.3573 \log _{2} 0.3573- \\
& 0.3573 \log _{2} 0.3573=2.3028
\end{aligned}
$$

\section{RESUMEN DE RESULTADOS}

En este trabajo se ha planteado un modelo para la estimación de los pe- 
sos de los componentes de la Gestión, y utilizado el concepto de Entropía de la información como una medida del desorden de los modelos de Gestión que existen en la actualidad.

\section{CONCLUSIONES}

Luego de la investigación desarrollada para aplicar la teoría matemática de la información a través de la entropía de Shannon a los sistemas de gestión, basándose en la topología de la configuración de los mismos, y a partir de los resultados obtenidos en el modelamiento y simulación, se alcanzan las siguientes conclusiones:

La entropía de la información constituye una medida de la incertidumbre que puede ser aplicada a los sistemas de gestión para medir el nivel de ordenamiento del sistema, siendo un sistema de gestión más ordenado aquel que obtenga la menor entropía posible y, en consecuencia, menos ordenado aquel que tenga una mayor entropía relativa.

Existe una convergencia definida de pesos específicos hacia un sistema de pesos ideales que reflejan el aporte particular de cada componente en la gestión, notándose un fuerte incremento de pesos en los componentes de priorización, creatividad, innovación y control gerencial como los componentes de mayor significancia y peso relativo.

El mecanismo de medición evidenciado puede servir como base de comparación para evaluar sistemas de gestión que pueden ser muy útiles al momento de considerar la compra o implantación de un sistema de gestión individual o integrado en una organización empresarial o industrial.

El modelo planteado para la estructura de pesos de los Modelos Integrados de Gestión es muy próximo a los pesos empíricos que la literatura reporta para estos. El concepto de Entropía de la información ha sido aplicado a estos Modelos Integrados de Gestión como un indicador de desorden, que se traduce como una menor efectividad del Modelo para la industria.

\section{Apéndice}

Si consideramos el problema con una solo restricción:

$$
\begin{aligned}
\text { Min } & \sum_{i=1}^{5} K_{i}\left(p_{i}-m_{i}\right)^{2} \\
\text { s.a } & \sum_{i=1}^{5} a_{i} p_{i}=\vec{a} \cdot \vec{p}=1 \\
& \vec{a}=(4,1,2,2,1
\end{aligned}
$$

Resolvemos usando los multiplicadores de Lagrange, y obtenemos:

Entonces, $\quad p_{i}=-\frac{a_{i} \lambda}{2 K_{i}}+m_{i} \ldots$ (A.2)

Reemplazamos en la ecuación (3)

$$
-\sum_{i=1}^{5} \frac{a_{i}{ }^{2} \lambda}{2 K_{i}}+a_{i} m_{i}=1
$$

Despejamos $\lambda$,

$$
\lambda=(-1+\vec{a} \cdot \vec{m})\left(\sum_{i=1}^{5} \frac{a_{i}^{2}}{2 K_{i}}\right)^{-1}
$$

Reemplazamos en la ecuación (A.2)

$$
p_{i}=\frac{a_{i}}{K_{i}}(-1+\vec{a} \cdot \vec{m})\left(\sum_{i=1}^{5} \frac{a_{i}^{2}}{2 K_{i}}\right)^{-1}+m_{i}
$$

$\mathrm{Y}$ podemos expresarlo por comodidad

$$
\begin{aligned}
& p_{i}=f_{i}(\vec{K})(-1+\vec{a} \cdot \vec{m}) a_{i}+m_{i} \\
& \text { donde, } \quad f_{i}(\vec{K})=\left(\sum_{i=1}^{5} \frac{a_{i}^{2}}{K_{i}}\right)^{-1} \frac{1}{K_{i}}
\end{aligned}
$$


Como se mencionó, las valores que pueden tomar $\rightarrow$ no son tan importantes; para poder mostrar esto, hallaremos el máximo de $\rightarrow$ derivando.

$$
\frac{d f_{i}}{d K_{i}}=-\left(\sum_{i=1}^{5} \frac{a_{i}^{2}}{K_{i}}\right)^{-2}\left(-\frac{a_{i}^{2}}{K_{i}^{3}}\right)+\left(\sum_{i=1}^{5} \frac{a_{i}^{2}}{K_{i}}\right)^{-1}\left(-\frac{1}{K_{i}^{2}}\right)
$$

Igualamos a $0, \frac{d f_{i}}{d K_{i}}=0$, entonces

$$
\begin{aligned}
& \left(\sum_{i=1}^{5} \frac{a_{i}^{2}}{K_{i}}\right)^{-1}\left(-\frac{a_{i}^{2}}{K_{i}^{2}}\right)=\frac{1}{K_{i}} \\
& K_{i}=a_{i}^{2}\left(\sum_{i=1}^{5} \frac{a_{i}^{2}}{K_{i}}\right)^{-1}
\end{aligned}
$$

Evaluamos,

$$
f_{i}^{\max }=\frac{1}{a_{i}^{2}}
$$

Resultado que no depende de los valores de $\rightarrow$. Esto nos permite arbitrariamente simular valores de $\rightarrow$, sin importarnos el rango en que se encuentren. Como los resultados obtenidos en 1, mostramos a continuación el histograma con valores de

Figura (4), donde se observa que los resultados son similares al presentado anteriormente, evidenciando que el rango de ${ }^{\rightarrow}$ no es significativo en la solución del modelo propuesto.
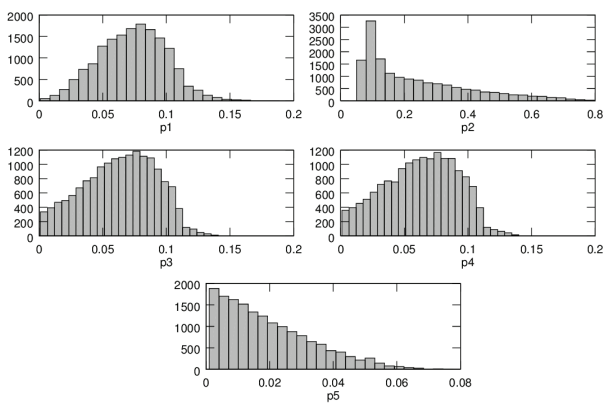

Figura 9. Histograma de los valores de $p_{i}$ con $0<K_{i}<1$

\section{REFERENCIAS BIBLIOGRÁFICAS}

1. Frizelle, G. \& Woodcock, E. (1995). "Measuring complexity as an aid to developing operational strategy". International Journal of Operations and Production Management Vol. 15, No 5, 26-39.

2. Isik, F. (2010). "An entropy-based approach for measuring complexity in supply chains". International Journal of Production Research Vol. 48, No 12, 3681-3696.

3. Kannan, S. M.; Jeevanantham, A. K. \& Jayabalan, V. (2008). "Modelling and analysis of selective assembly using Taguchi's loss function". International Journal of Production Research, Vol 46, No 15, 4309-4330.

4. Liu, Peide \& Zhang, Xin. (2011). "Research on the supplier selection of a supply chain based on entropy weight and improved ELECTREIII method". International Journal of Production Research Vol. 49 No 3, 637-646 .

5. Shannon, E. (1948). "A Mathematical Theory of Communication”. The Bell System Technical Journal, Vol. 27, 379-423, 623- 656.

6. OHSAS 18001:2007. (2007). Occupational health and safety management systems-specification, BSI: British Standard Institution. 
7. Chan, Joseph O. (2009). "A Conceptual Framework for an Integrated Knowledge-Driven Enterprise Model”. Journal of International Technology and Information Management, Vol. 18 No 2, 161-185.

8. Parr, Anne \& Shanks, Graeme. (2000). "A model of ERP project implementation”. Journal of Information Technology Vol. 15, 289- 303.

9. ISO 9001:2008, (2008). Quality managment system. Requirements, ISO.

10. Baldrige. (27/04/2012). Performance Excellence Program. National Institute of Standards and Technology. http:/ / www.nist.gov/baldrige/

11. Sarmiento, Ana \& Rakesh, Nagiy. (1999) "A Review of Integrated Analysis of Production-Distribution Systems”. IIE Trans. N_31, 1061-1074.

12. Bernardo, M.; Casadesus, M.; Karapetrovic, S. \& Heras, I. (2012). "Do integration difficulties influence management system integration levels?”. Journal of Cleaner Production Vol. 21, 23-33.

13. Rocha, M.; Searcy, C. \& Karapetrovic, S. (2007). "Integrating Sustainable Development into Existing Management Systems". Total Quality Management Vol. 18, Nos. 1-2, 83-92.

14. Isik, F. (2010). "An entropy-based approach for measuring complexity in supply chains". International Journal of Production Research Vol. 48, No 12, 3681-3696.

15. Hendricks K. B. \& Singhal, V. R. (2011). "The effect of supply chain glitches on shareholder wealth". Journal of Operations Management Vol. 21, 501-522.

16. Asif, M.; Bruijn, E.; Fisscher, O. \& Pagell, M. (2010). "Integration of management systems: A methodology for operational excellence and strategic flexibility”. Operations Management Research Vol. 3 No 3-4, 146-160.

17. Asif, M.; Fisscher, Searcy C. \& Zutshi, A. (2011). "An integrated management systems approach to corporate social responsibility". Journal of Cleaner Production. Available online 2.

18. Elbashir, M. Z.; Collier, P. A. \& Sutton, S. G. (2011). "The Role of Organizational Absorptive Capacity in Strategic Use of Business Intelligence to Support Integrated Management Control Systems". The Accounting Review. Vol. 86, N1, 155-184.

19. Prybutok, V. R; Richards, R. M. \& Cutshall, R. (2001). "The significance of information and analysis as a component of a leadership model based on Malcolm Baldrige National Quality Award Criteria”. The Journal of Computer Information Systems Vol.41 N4, 52-56.

20. Weiming, S., Qi, H. \& Yunjiao, X. (2012). "A loosely coupled system integration approach for decision support in facility management and maintenance". Automation in Construction Vol. 25, 41-48.

21. Grabot, B.; Mayere, A. \& Bazet, I. (2008). "ERP Systems and Organisational Change: A Socio-technical Insight”. Springer VerlagLondon-Reino Unido.

22. Chan, J. O. (2010). "E-Business Enabled ERP II Architecture”. Communications of the IIMAc 2010, Volume 10 Issue 1. 